ARTICLE

Received 16 Aug 2016 | Accepted 5 Dec 2016 | Published 24 Jan 2017 DOl: 10.1038/ncomms14183 OPEN

\title{
Drug regimens identified and optimized by output-driven platform markedly reduce tuberculosis treatment time
}

Bai-Yu Lee ${ }^{1, \star}$, Daniel L. Clemens ${ }^{1, \star}$, Aleidy Silva², Barbara Jane Dillon', Saša Masleša-Galić ${ }^{1}$ Susana Nava1, Xianting Ding ${ }^{3}$, Chih-Ming Ho ${ }^{2,4} \&$ Marcus A. Horwitz ${ }^{1}$

The current drug regimens for treating tuberculosis are lengthy and onerous, and hence complicated by poor adherence leading to drug resistance and disease relapse. Previously, using an output-driven optimization platform and an in vitro macrophage model of Mycobacterium tuberculosis infection, we identified several experimental drug regimens among billions of possible drug-dose combinations that outperform the current standard regimen. Here we use this platform to optimize the in vivo drug doses of two of these regimens in a mouse model of pulmonary tuberculosis. The experimental regimens kill $M$. tuberculosis much more rapidly than the standard regimen and reduce treatment time to relapse-free cure by $75 \%$. Thus, these regimens have the potential to provide a markedly shorter course of treatment for tuberculosis in humans. As these regimens omit isoniazid, rifampicin, fluoroquinolones and injectable aminoglycosides, they would be suitable for treating many cases of multidrug and extensively drug-resistant tuberculosis.

\footnotetext{
${ }^{1}$ Division of Infectious Diseases, Department of Medicine, University of California, Los Angeles, California 90095, USA. ${ }^{2}$ Department of Mechanical and Aerospace Engineering, University of California, Los Angeles, California 90095, USA. ${ }^{3}$ Med-X Research Institute, School of Biomedical Engineering, Shanghai Jiao Tong University, Shanghai 200030, China. ${ }^{4}$ Department of Bioengineering, University of California, Los Angeles, California 90095, USA. * These authors contributed equally to this work. Correspondence and requests for materials should be addressed to M.A.H. (email: MHorwitz@mednet.ucla.edu).
} 
T uberculosis (TB) is a major health problem of global proportions. In 2014, an estimated 9.6 million people fell ill with $\mathrm{TB}$ and 1.5 million people died, placing TB above $\mathrm{HIV}$ as the number one infectious cause of death worldwide ${ }^{1}$. Although drug-sensitive TB, which comprises $96.7 \%$ of new cases, is a treatable disease, the current standard treatment requires 6-8 months of a multi-drug regimen to achieve relapse-free cure $^{2,3}$. This long course of treatment is often associated with toxicity, poor adherence and development of drug resistance. A more effective drug combination that provides more rapid sterilization of tissues has the potential to ameliorate these critical problems ${ }^{4-6}$.

History suggests that the long duration of treatment required to achieve relapse-free cure may be attributable more to limitations in bactericidal activity of the antimicrobial drug combination than to the intrinsic biology of Mycobacterium tuberculosis. The first regimen in 1952, consisting of streptomycin, isoniazid (INH) and para-aminosalicylic acid required 24 months therapy; replacing para-aminosalicylic acid by ethambutol (EMB) in the 1960s and subsequently adding rifampicin (RIF) in the 1970s sequentially shortened treatment duration first to 18 months and then to 9-12 months, and substituting pyrazinamide (PZA) for streptomycin in the $1980 \mathrm{~s}$ further cut treatment duration to 6-8 months ${ }^{7}$.

These previous treatment regimens, culminating in today's Standard Regimen (INH, RIF, EMB and PZA), in which one drug was added or substituted at a time, were not based on a systematic search to identify the most highly synergistic drug combinations. Indeed, contrary to the concept of synergy, two of the four drugs in the Standard Regimen (EMB and PZA) are dropped after 2 months to reduce patient exposure to potential toxic side effects, as by this point they are considered superfluous to treatment and no longer needed to prevent the emergence of drug resistance. Thus, it is likely that an approach centred on identifying the most highly synergistic drug combinations has the potential to shorten further the duration of treatment required to achieve relapse-free cure of $\mathrm{TB}$.

Since conventional evaluation of a large number of TB drugs, even at a few doses, would require testing of an enormous number of drug-dose combinations, a systematic approach is required to identify the most promising drug combinations for treating TB. We have previously developed a method, called Feedback System Control, Second Generation (FSC.II) or Parabolic Response Surface (PRS), in which experimental data is used to solve the second order algebraic equation that describes the drug dose-efficacy response of a multi-drug combination. The PRS approach can map out the entire drug dose-efficacy landscape from a very large search space and rapidly home in on optimal drug-dose combinations. In previous work, PRS enabled us to identify numerous combinations of TB drugs that exhibit higher antimicrobial activity than the Standard Regimen (INH, RIF, EMB and PZA) in vitro in a human macrophage model of $M$. tuberculosis infection ${ }^{8}$. Both fluorescence-based bacterial metabolic activity and confirmatory CFU-based killing studies identified two regimens as having greater efficacy than the Standard Regimen-PRS Regimen I (Clofazimine (CLZ)/EMB/ Prothionamide (PRO)/PZA) and PRS Regimen II (CLZ/EMB/ Bedaquiline (BDQ)/PZA), in which PRO was replaced with the recently developed drug, BDQ. However, preclinical studies in animal models are essential to evaluating the safety and efficacy of new multidrug regimens and determining which combinations merit advancement to clinical trials ${ }^{9}$. While no animal model is perfect, the $\mathrm{BALB} / \mathrm{c}$ mouse model of pulmonary $\mathrm{TB}$ has been broadly predictive of human clinical results and provides a practicable model for comparing multiple TB drug regimens ${ }^{10-12}-$ such as those that we identified using the PRS method in vitro.
Here, using the mouse model, we apply the PRS method to model the parabolic drug dose-efficacy response surface ${ }^{13-20}$ of two of the regimens and thereby identify optimal drug doses for use of these regimens in vivo. Since in vivo and in vitro drug dose ratios can differ markedly, dose re-optimization is a crucial step for ensuring the success of in vivo studies. As the PRS approach to transitioning from in vitro to in vivo drug doses is output-driven, it is agnostic to such considerations as drug mechanism and bacterial metabolic state, automatically takes into account drug-drug interactions, and is not dependent on pharmacokinetic studies. We then compare the two optimized experimental regimens with the Standard Regimen for time required to attain culture negativity in infected lung tissues and to achieve relapse-free cure.

\section{Results}

Efficacy study of PRS Regimen I. To determine the optimal dose for individual drugs in PRS Regimen I, we mapped the drug dose-efficacy response surface after a 4-week course of treatment in a mouse model of pulmonary TB. For each drug, the highest dose used was equal to the highest dose previously used in mouse models of pulmonary $\mathrm{TB}$ in the literature, and the dose range chosen included the standard human equivalent dose. To reduce the number of different treatment groups required to map the drug dose-efficacy response surface with PRS methodology to a practicable number (10 groups), we held the dose of one (CLZ) of the 4 drugs constant, while varying the doses of the other three drugs. We infected mice by aerosol with the highly virulent M. tuberculosis strain Erdman, delivering 2.22 \pm 0.09 (mean \pm s.e.m.) $\log _{10}$ colony forming units (CFU) of $M$. tuberculosis per lung. Higher challenge doses, such as those used by others ${ }^{10}$ in similar types of studies employing the less virulent $M$. tuberculosis strain H37Rv (ref. 21), could not be used because mice challenged with such doses of the Erdman strain died before treatment could be initiated at two-weeks post-challenge. By two weeks post challenge, the burden of $M$. tuberculosis had increased by 4 logs to $6.24 \pm 0.05 \log _{10} \mathrm{CFU}$ per lung, at which point we initiated antibiotic treatment by oral gavage five times a week (Monday-Friday). Ten groups of mice received CLZ at $25 \mathrm{mg} \mathrm{kg}^{-1}$ and EMB, PRO and PZA were given at permutations of high, middle (1/3rd the high dose) or low (1/9th the high dose) doses as indicated in Table 1; Fig. 1a. As controls, one group of mice was treated with the Standard Regimen (INH/RIF/EMB/PZA) and another group received sham treatment.

On completion of the 4-week treatment, the sham-treated mice had $6.67 \pm 0.09 \log _{10}$ CFU of $M$. tuberculosis in their lungs (Fig. 1a; Supplementary Table 1). Treatment with the Standard Regimen significantly reduced lung burden by $2.43 \log _{10} \mathrm{CFU}$ $(P<0.0001$, one-way ANOVA with Tukey's correction). Mice in all 10 PRS Regimen I-treated groups had fewer CFU in their lungs than mice treated with the Standard Regimen. The best PRS Regimen I group (that is $\mathrm{HHHH}$ ) had 1.79 logs fewer CFU in their lungs than mice treated with the Standard Regimen $(P<0.0001$, one-way ANOVA with Tukey's correction). These results correlated with the severity of gross pathology and number of tubercles on the lung surface (Fig. 2). Thus, PRS Regimen I has markedly greater bactericidal activity in the mouse model than the Standard Regimen. On completion of the 4-week treatment, the sham-treated mice had $4.07 \pm 0.19 \log _{10} \mathrm{CFU}$ of M. tuberculosis in the liver and $5.03 \pm 0.11 \log _{10}$ CFU in the spleen, while all treated groups had $<2.2 \log _{10}$ CFU in the liver and less than $1.7 \log _{10} \mathrm{CFU}$ in the spleen.

A parabolic drug dose-efficacy response surface was generated from the regression equation fitted to the lung $\log _{10}$ CFU data 


\begin{tabular}{|c|c|c|c|c|}
\hline \multirow{2}{*}{$\begin{array}{l}\text { Treatment } \\
\text { Control Standard Regimen }\end{array}$} & \multicolumn{4}{|c|}{ Drug dose $\left(\mathrm{mg} \mathrm{kg}^{-1}\right)$} \\
\hline & $\mathrm{INH}$ & RIF & EMB & PZA \\
\hline Standard Regimen & 25 & 10 & 100 & 150 \\
\hline Enhanced Standard Regimen & 25 & 10 & 100 & 450 \\
\hline $\begin{array}{l}\text { Experimental TB Drug Regimens } \\
\text { PRS Regimen I }\end{array}$ & CLZ & $\mathrm{EMB}$ & PRO & PZA \\
\hline $\mathrm{H} / \mathrm{L} / \mathrm{L} / \mathrm{H}$ & 25 & 11.1 & 8.3 & 450 \\
\hline $\mathrm{H} / \mathrm{L} / \mathrm{H} / \mathrm{L}$ & 25 & 11.1 & 75 & 50 \\
\hline $\mathrm{H} / \mathrm{H} / \mathrm{L} / \mathrm{L}$ & 25 & 100 & 8.3 & 50 \\
\hline $\mathrm{H} / \mathrm{H} / \mathrm{H} / \mathrm{H}$ & 25 & 100 & 75 & 450 \\
\hline $\mathrm{H} / \mathrm{H} / \mathrm{H} / \mathrm{L}$ & 25 & 100 & 75 & 50 \\
\hline $\mathrm{H} / \mathrm{H} / \mathrm{L} / \mathrm{H}$ & 25 & 100 & 8.3 & 450 \\
\hline $\mathrm{H} / \mathrm{L} / \mathrm{H} / \mathrm{H}$ & 25 & 11.1 & 75 & 450 \\
\hline $\mathrm{H} / \mathrm{H} / \mathrm{M} / \mathrm{M}$ & 25 & 100 & 25 & 150 \\
\hline $\mathrm{H} / \mathrm{M} / \mathrm{H} / \mathrm{M}$ & 25 & 33.3 & 75 & 150 \\
\hline \multirow[t]{2}{*}{$\mathrm{H} / \mathrm{M} / \mathrm{M} / \mathrm{H}$} & 25 & 33.3 & 25 & 450 \\
\hline & CLZ & $E M B$ & $\mathrm{BDQ}$ & PZA \\
\hline \multicolumn{5}{|l|}{ PRS Regimen II } \\
\hline $\mathrm{H} / \mathrm{L} / \mathrm{L} / \mathrm{H}$ & 25 & 11.1 & 5.6 & 450 \\
\hline $\mathrm{H} / \mathrm{L} / \mathrm{H} / \mathrm{L}$ & 25 & 11.1 & 50 & 50 \\
\hline $\mathrm{H} / \mathrm{H} / \mathrm{L} / \mathrm{L}$ & 25 & 100 & 5.6 & 50 \\
\hline $\mathrm{H} / \mathrm{H} / \mathrm{H} / \mathrm{H}$ & 25 & 100 & 50 & 450 \\
\hline $\mathrm{H} / \mathrm{H} / \mathrm{H} / \mathrm{L}$ & 25 & 100 & 50 & 50 \\
\hline $\mathrm{H} / \mathrm{H} / \mathrm{L} / \mathrm{H}$ & 25 & 100 & 5.6 & 450 \\
\hline $\mathrm{H} / \mathrm{L} / \mathrm{H} / \mathrm{H}$ & 25 & 11.1 & 50 & 450 \\
\hline $\mathrm{H} / \mathrm{H} / \mathrm{M} / \mathrm{M}$ & 25 & 100 & 16.7 & 150 \\
\hline $\mathrm{H} / \mathrm{M} / \mathrm{H} / \mathrm{M}$ & 25 & 33.3 & 50 & 150 \\
\hline $\mathrm{H} / \mathrm{M} / \mathrm{M} / \mathrm{H}$ & 25 & 33.3 & 16.7 & 450 \\
\hline PRS Regimen IIA & 25 & 100 & 16.7 & 150 \\
\hline PRS Regimen IIB & 25 & 100 & 16.7 & 450 \\
\hline PRS Regimen IIC & 25 & 100 & 30 & 450 \\
\hline
\end{tabular}

$\mathrm{BDQ}$, Bedaquiline; $\mathrm{CLZ}$, Clofazimine; $\mathrm{EMB}$, Ethambutol; $\mathrm{H}$, high dose; INH, Isoniazid; L, low dose; $\mathrm{M}$, middle dose; PRO, Prothionamide; PZA, Pyrazinamide; RIF, Rifampicin.

from the 10 groups of mice treated with various drug-dose combinations of PRS Regimen I (Methods, equation 2). This drug dose-efficacy response surface had a fitting correlation $(r)$ of 0.999 between the measured $\log _{10}$ CFU and the projected $\log _{10} \mathrm{CFU}$ and an adjusted $R^{2}$ value of 0.993 indicating that the model explains $99.3 \%$ of the variability in the projected $\log _{10}$ CFU. A comparison of the observed means versus the predicted means of the response surface model showed a per cent difference \{per cent difference $=100 \times($ observed mean-predicted mean $) /$ ((observed mean + predicted mean $) / 2)\}$ of $\leq 1.4 \%$ with an average difference of $0.036 \%$. Levene's test showed no deviation from homoscedasticity among the 10 PRS Regimen I s.d., satisfying the requirements for s.d. pooling in the parabolic and ANOVA analyses.

We found that, with CLZ and PZA doses kept constant, the drug dose-response surface for EMB and PRO was relatively flat, as dose variations of these two drugs had relatively little influence on the efficacy of PRS Regimen I to reduce the lung burden of M. tuberculosis (Fig. 1c, top). In contrast, differences in PZA dose had a major impact on lung burden reduction of the combination regimen (Fig. 1c, middle and bottom). Among the 10 PRS Regimen I-treated groups, mice treated with the individual drugs at their highest dose had the lowest lung burden of M. tuberculosis, whereas mice treated with the identical drug doses except for PZA, which was at low dose, had the highest lung bacterial burden. The difference in bacterial burden in the lungs between these two groups was highly significant $(P<0.0001$, one-way ANOVA with Tukey's correction).
Thus, both the drugs and the drug ratio in a drug combination are important to the outcome of the treatment.

Efficacy study of PRS Regimen II. We applied the same strategy to map the drug dose-efficacy response surface and assess treatment efficacy for PRS Regimen II (Fig. 1b; Supplementary Table 2). Mice that were sham-treated had $6.71 \pm 0.05 \log _{10}$ CFU of M. tuberculosis per lung at the end of the 4-week treatment period (Supplementary Table 2). Treatment with the Standard Regimen reduced lung burden of $M$. tuberculosis by 2.77 logs $(P<0.0001$, one-way ANOVA with Tukey's correction). Mice treated with any of the 10 dose variations of the PRS Regimen II (with various doses of EMB, BDQ and PZA) reduced bacterial burden in the lung by 4.75 to 5.95 logs to a level significantly lower than that achieved by the Standard Regimen $(P<0.0001$, one-way ANOVA with Tukey's correction). Mice in the best PRS Regimen II-treated group had 3.2 logs fewer CFU in their lung than mice treated with the Standard Regimen. Mice treated with PRS Regimen II also exhibited the least gross lung pathology and number of tubercles on the lung compared with mice that were sham-treated or treated with the Standard Regimen (Fig. 3). Thus, PRS Regimen II is significantly more efficacious than the Standard Regimen. PRS Regimen II is also more potent than PRS Regimen I, as indicated by a larger reduction in lung burden; indeed, in 4 out of 10 groups, no organism was detected in any of the animals. These two regimens differ by a single drug with PRO in PRS Regimen I replaced by BDQ in PRS Regimen II. With $\mathrm{BDQ}$, instead of $\mathrm{PRO}$ in the regimen, variation of the PZA dose had a smaller impact on treatment efficacy (Fig. 1d, middle and bottom). However, decreasing the dose of BDQ alone or that of BDQ and PZA together adversely affected the efficacy of PRS Regimen II (Fig. 1d, bottom).

On the basis of lung $\log _{10}$ CFU data, a parabolic drug doseefficacy response surface was generated for PRS Regimen II which had a fitting correlation of 0.995 and an adjusted $R^{2}$ value of 0.981 (Methods, equation 3). A comparison of the observed means versus the predicted means of the response surface model showed a per cent difference of $\leq 6.8 \%$ with an average difference of $0.33 \%$. One drug combination group (that is, $\mathrm{HMMH}$ ) among the 10 PRS Regimen II groups was identified as an outlier under the model and was omitted in a repeat assessment. The model estimated that optimal doses of the drugs in the combination were about the same with or without this group in the model. Levene's test showed no deviation from homoscedasticity among 9 of the 10 PRS Regimen II s.d., satisfying the requirements for s.d. pooling in the parabolic and ANOVA analyses.

PRS regimens rapidly render lungs culture negative. To determine the treatment time required for the PRS regimens to render the lung of $M$. tuberculosis-infected mice culture negative, we conducted a direct comparison of the treatment efficacy of these two PRS regimens against the Standard Regimen (Tables 2 and 3). Since the lung burden of M. tuberculosis in PRS Regimen II-treated mice in the 4-week short-term study was at the experimental limit of detection when PZA was administered at both the low and high doses, we assessed PRS Regimen II in an 8 -week treatment study with PZA both at the $150 \mathrm{mg} \mathrm{kg}^{-1}$ middle dose and the $450 \mathrm{mg} \mathrm{kg}^{-1}$ high dose, which we termed PRS Regimen IIA and IIB, respectively (Fig. 4; Table 1). In addition, we included in this study the Enhanced Standard Regimen, where the dose of PZA was increased to $450 \mathrm{mg} \mathrm{kg}^{-1}$, the same as in the optimized PRS Regimen I. Although the $150 \mathrm{mg} \mathrm{kg}^{-1}$ dose of PZA is commonly used in mouse studies $^{22,23}$ and is thought to match the standard human dose, a higher $300 \mathrm{mg} \mathrm{kg}^{-1}$ dose of PZA has been shown to have 
a

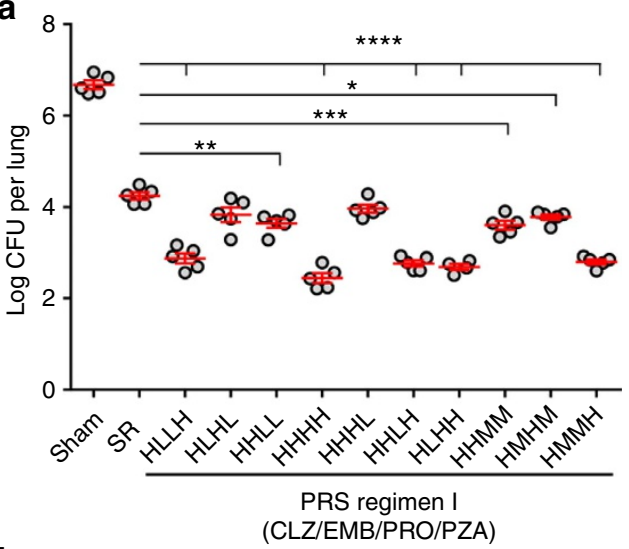

b

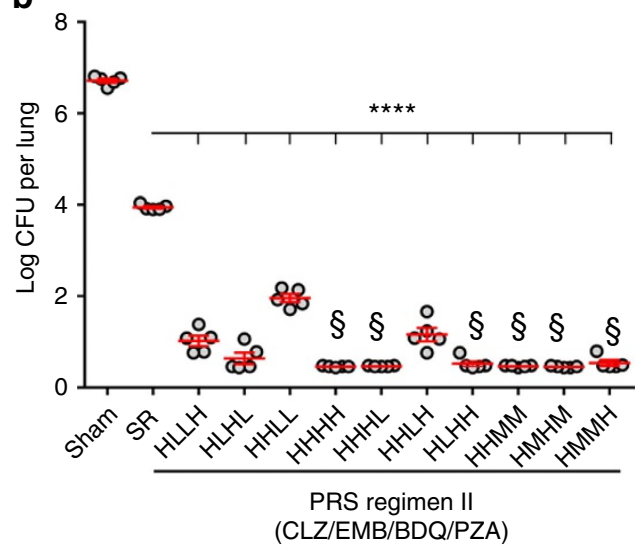

CLZ/EMB/BDQ/PZA) c
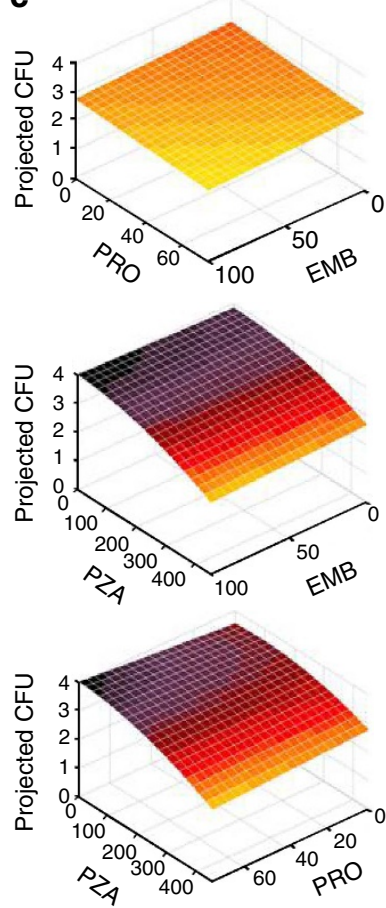

d
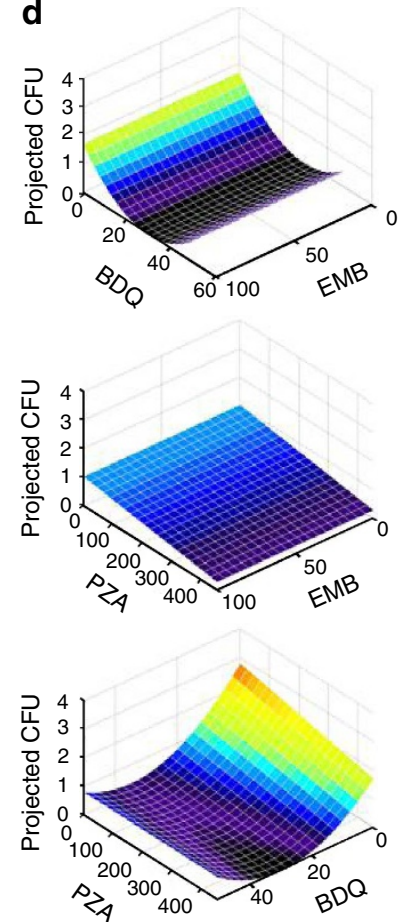

0
2.5

Figure 1 | Short-term efficacy studies of PRS Regimens I and II. $(\mathbf{a}, \mathbf{b})$ Lung burden of $M$. tuberculosis in mice that were sham-treated, treated with the Standard Regimen (SR) or treated with PRS Regimens I (a) or II (b) with the drugs administered at high (H), middle (M) or low (L) dose five times per week for 4 weeks. Data are mean \pm s.e.m. of $\log _{10}$ CFU for $n=5$ mice per group. All treatment groups had significantly fewer CFU than the sham-treated group $(P<0.0001)$. Differences in treatment efficacy between the Standard Regimen and individual PRS Regimen I or II groups were evaluated by one-way ANOVA with Tukey's correction. ${ }^{\star} P<0.05,{ }^{\star \star} P<0.01,{ }^{\star \star \star} P<0.001,{ }^{\star \star \star \star} P<0.0001$. ${ }^{\S}$ No $M$. tuberculosis CFU detected-data plotted as discussed in Methods. (c,d) Heat maps of the drug-dose response surface for PRS Regimens I (c) and II (d). These 3-dimensional graphs show how the projected number of lung CFU changes as the dose of one and/or the other drug is increased or decreased. Drug dose is shown in $\mathrm{mg} \mathrm{kg}^{-1}$. In these plots, in addition to $C L Z$, the third drug is kept at the high dose.

greater efficacy in both mouse and guinea pig $^{24}$ and the addition of a $450 \mathrm{mg} \mathrm{kg}^{-1}$ dose of PZA has been shown to increase the efficacy of a gatifloxacin, ethionamide combination in a mouse model of pulmonary TB (ref. 25).

In this 8-week treatment study, at the time treatment was started two weeks post-challenge, the number of $M$. tuberculosis CFU per lung in infected mice was $6.04 \pm 0.08 \log _{10}$ (Fig. 4a; Supplementary Table 3). Without treatment, M. tuberculosis continued to multiply, and by two weeks later, the lung burden of M. tuberculosis plateaued at $\sim 7 \log _{10}$ CFU for the duration of the experiment. Treatment with the Standard Regimen reduced M. tuberculosis burden in the lung by 2.6 logs at week 2 and by 5.2 logs at week 8 . Although the difference was not statistically significant at week 3, the Enhanced Standard Regimen was significantly more efficacious than the Standard Regimen at week $4(P<0.001)$ and week $8(P<0.0001$, one-way ANOVA with Tukey's correction). At the end of the 8 -week treatment study, $M$. tuberculosis could still be cultured from the lungs of mice treated with the Standard Regimen and Enhanced Standard Regimen. Indeed, in a subsequent 20 -week long-term treatment study, we found that it took 16 weeks for the Standard Regimen and 12 weeks for Enhanced Standard Regimen to attain culture negativity in the lungs (Fig. 5a; Supplementary Table 4a).

As in the short-term study, bacterial load in the lungs of mice treated with PRS Regimen I was significantly less than that of the
Standard Regimen at week $4(P<0.0001$, one-way ANOVA with Tukey's correction; Fig. 4a,c). Moreover, throughout the 8-week treatment period, lung CFU counts in mice treated with PRS Regimen I was lower than in mice treated with the Enhanced Standard Regimen; in both of these regimens, PZA was at the same dose of $450 \mathrm{mg} \mathrm{kg}^{-1}$. The time to attain lung culture negativity for mice treated with PRS Regimen I was 8 weeks, a much shorter duration than the 16 and 12 weeks of treatment required for the Standard Regimen and the Enhanced Standard Regimen, respectively (Fig. 5a; Supplementary Table 4a).

Both PRS Regimen IIA and IIB killed M. tuberculosis at a rate much faster than the Standard regimen, Enhanced Standard Regimen, and PRS Regimen I. While treatment with the Standard Regimen reduced lung burden of $M$. tuberculosis by 4 logs at week 6 and by 5 logs at week 8, treatment with PRS Regimen II achieved these levels at week 2 and 3, respectively (Fig. 4c). Thus, PRS Regimen II is superior to PRS Regimen I, and PRS Regimen I is significantly better than the Standard Regimen and the Enhanced Standard Regimen.

The drug dose-efficacy response surface generated for BDQ and PZA predicts a higher treatment efficacy for PRS Regimen II with BDQ at $30 \mathrm{mg} \mathrm{kg}^{-1}$ and PZA at $450 \mathrm{mg} \mathrm{kg}^{-1}$; this regimen was designated as PRS Regimen IIC (Fig. 5b; Table 1). Treatment with PRS Regimen IIC 5 days per week for merely 3 weeks reduced the number of $M$. tuberculosis to 1 organism per lung on 
Sham

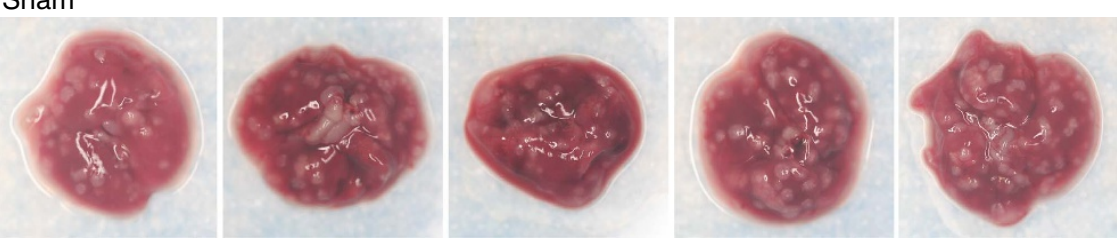

Standard regimen
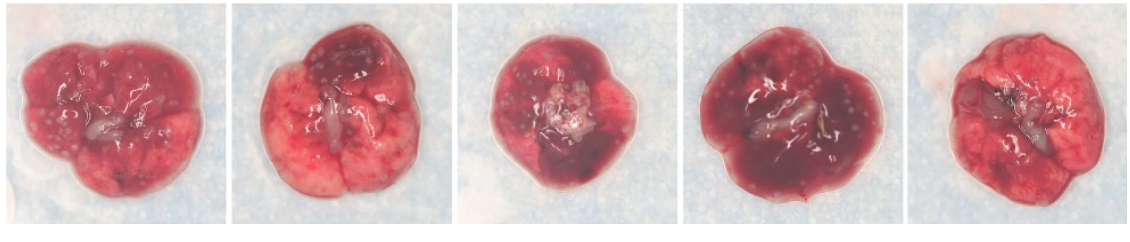

PRS regimen I (CLZ/EMB/PRO/PZA)

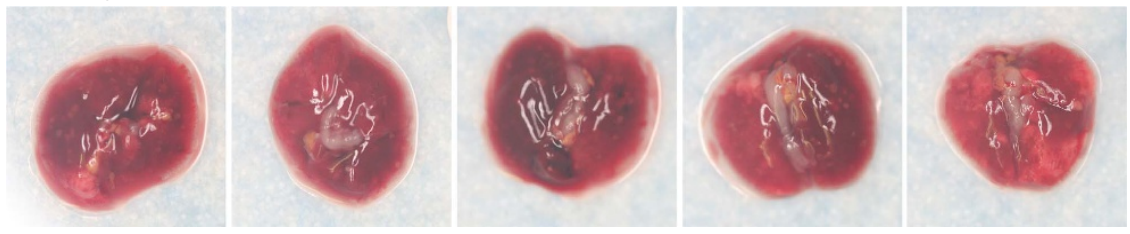

Figure 2 | Lung pathology of mice that received sham or Standard Regimen or PRS Regimen I treatment. Shown are representative gross pathology images of lungs dissected from mice that were sham-treated or treated with the Standard Regimen or PRS Regimen I at high doses of each drug by oral gavage five times (Monday-Friday) a week for 4 weeks.
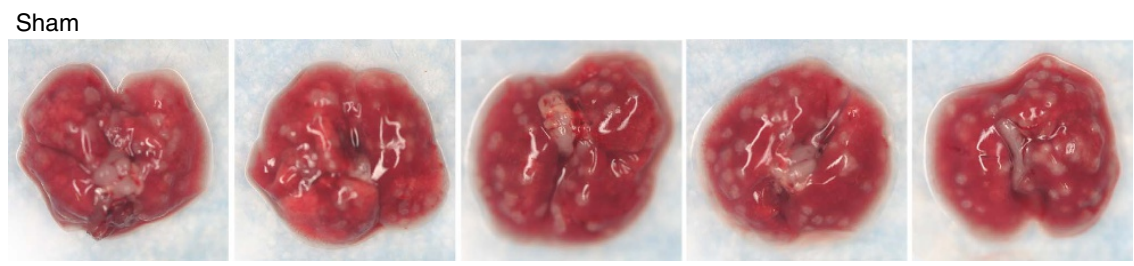

\section{Standard regimen}
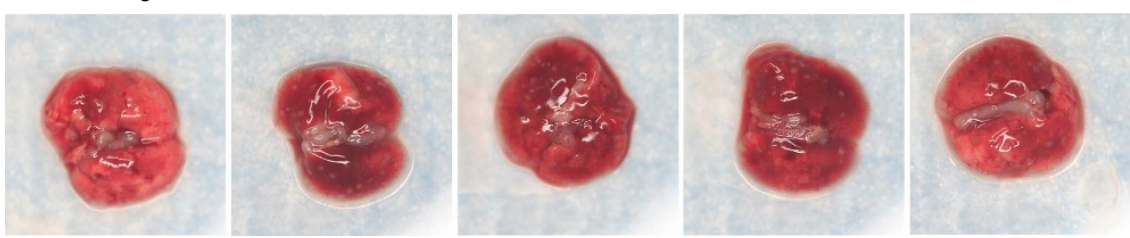

PRS regimen II (CLZ/EMB/BDQ/PZA)
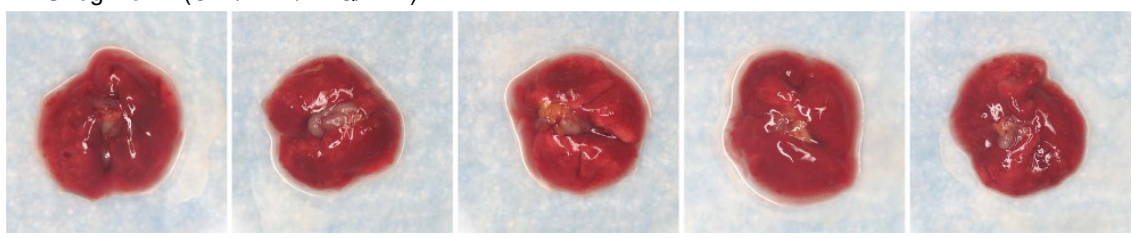

Figure 3 | Lung pathology of mice that received sham or Standard Regimen or PRS Regimen II treatment. Shown are representative gross pathology images of lungs dissected from mice that were sham-treated or treated with the Standard Regimen or PRS Regimen II at high doses of each drug by oral gavage five times (Monday-Friday) a week for 4 weeks.

average with $40 \%$ of the mice ( 2 out of 5 mice) culture negative in their lungs. Daily treatment for 3 weeks led to lung culture negativity in $80 \%$ of the mice ( 4 out of 5 mice) (Fig. 5a; Supplementary Table 4a).

PRS regimens rapidly cure mice. To assess relapse after treatment, we held mice for an additional 3 months after the completion of their antibiotic treatment, at which time they were killed and their entire lungs and spleens plated for CFU (Supplementary Table 4b-f). Relapse was defined as the finding of
1 or more CFU in the lung or spleen; however, lung counts alone would have sufficed to define relapse as all relapsed animals had at least $1 \mathrm{CFU}$ in the lung, and all of these mice had fewer or no CFU in the spleen. For the Standard Regimen, it took 16 weeks of treatment to clear the infection in the lungs and spleen and to achieve $100 \%$ relapse-free cure (Fig. 5c; Supplementary Table $4 \mathrm{~b}$ ). In contrast, for PRS Regimen I, relapse-free cure was achieved in $80 \%$ of mice (that is, 4 out of 5 mice) after 8 weeks treatment and $100 \%$ of mice after 12 -weeks treatment $(P=0.002$ versus Standard Regimen, log rank test) (Fig. 5c; Supplementary Table 4d). Treatment with PRS Regimen IIA 5 days per week 
Table 2 | Scheme of medium-term efficacy and relapse study.

\begin{tabular}{|c|c|c|c|c|c|c|c|c|}
\hline \multirow[t]{2}{*}{ Treatment $^{\dagger}$} & \multirow[t]{2}{*}{ Mice } & \multicolumn{7}{|c|}{$\begin{array}{c}\text { Week } \\
\text { (Number of mice killed per group) }^{\star}\end{array}$} \\
\hline & & -2 & 0 & 2 & 3 & 4 & 6 & 8 \\
\hline Sham & 25 & & & 5 & 5 & 5 & 5 & 5 \\
\hline Standard Regimen & 25 & & & 5 & 5 & 5 & 5 & 5 \\
\hline Enhanced Standard Regimen & 15 & & & & 5 & 5 & & 5 \\
\hline PRS Regimen I & 15 & & & & & 5 & 5 & 5 \\
\hline PRS Regimen IIB & 10 & & & 5 & 5 & & & \\
\hline
\end{tabular}

\begin{tabular}{|c|c|c|c|c|c|c|c|c|c|c|c|}
\hline Untreated & 8 & 3 & 5 & & & & & & & & \\
\hline Sham & 35 & & & & 5 & 5 & 5 & 5 & 5 & 5 & 5 \\
\hline Standard Regimen & 50 & & & & 5 & 5 & 5 & 5 & $\begin{array}{l}5 \\
5^{\ddagger}\end{array}$ & $\begin{array}{l}5 \\
5^{\ddagger}\end{array}$ & $\begin{array}{l}5 \\
5^{\ddagger}\end{array}$ \\
\hline PRS Regimen I & 20 & & & & & & & $\begin{array}{l}5 \\
5^{\ddagger}\end{array}$ & $\begin{array}{l}5 \\
5^{\ddagger}\end{array}$ & & \\
\hline PRS Regimen IIC & 30 & & & & $\begin{array}{l}5 \\
5^{\ddagger}\end{array}$ & $\begin{array}{l}5 \\
5^{\ddagger}\end{array}$ & $\begin{array}{l}5 \\
5^{\ddagger}\end{array}$ & & & & \\
\hline PRS Regimen IIC daily & 20 & & & $\begin{array}{l}5 \\
5^{\ddagger}\end{array}$ & $\begin{array}{l}5 \\
5^{\ddagger}\end{array}$ & & & & & & \\
\hline
\end{tabular}

achieved relapse-free cure in $63 \%$ of mice at 3 weeks and $100 \%$ at 4 weeks (Fig. $4 \mathrm{~b}$ and Supplementary Table 3). Treatment with PRS Regimen IIC 5 days per week achieved relapse-free cure in $80 \%$ of mice at 3 weeks and $100 \%$ at 4 weeks (Fig. 5c; Supplementary Table 4e). Treatment with PRS Regimen IIC daily further accelerated relapse-free cure, achieving relapse-free cure in $40 \%$ of mice by 2 weeks and $100 \%$ by three weeks (Fig. $5 \mathrm{c}$; Supplementary Table 4f). Thus, compared with the Standard Regimen, PRS Regimen IIC, when administered at the same frequency (5 days per week), reduces the duration of treatment required to achieve $80 \%$ relapse-free cure from 12 weeks to 3 weeks and $100 \%$ relapse-free cure from 16 weeks to 4 weeks $(P<0.0001$ versus Standard Regimen, log rank test), a $75 \%$ reduction in time to relapse-free cure compared with the Standard Regimen. The difference in time to relapse-free cure between PRS Regimens I and IIC was highly statistically significant $(P<0.0001, \log$ rank test $)$.

\section{Discussion}

In this study, we employed PRS methodology to re-optimize the doses of two promising TB drug regimens in vivo, and we then evaluated the optimized regimens using a mouse model of pulmonary TB. Dose re-optimization by PRS methodology, as opposed to the conventional approach of dose scaling, can avoid failures that may occur in transitioning from in vitro to in vivo studies. Because PRS methodology is output driven, that is, only the phenotypic response-the final outcome of drug treatment (CFU of $M$. tuberculosis in the lung in this case)-is measured experimentally, it is agnostic to drug mechanism and such considerations as the metabolic state of the bacteria. Moreover, the experimentally calibrated drug dose-efficacy response surface automatically allows for drug-drug interactions; hence, pharmacokinetic studies are not necessary to determine the optimal drug doses. Thus, this approach describes the optimal drug doses for achieving the desired endpoint (maximum efficacy-reflected by the lowest $\log _{10} \mathrm{CFU}$ in the lung in this case) without regard to the biological mechanisms that result in this outcome.

Both regimens comprised previously approved drugs and the first regimen (PRS Regimen I) comprised exclusively generic drugs. Both regimens were markedly more efficacious than the Standard Regimen in reducing the lung burden of $M$. tuberculosis. For example, at four weeks after the start of treatment, mice treated with PRS Regimen I had 1.3 logs fewer CFU in their lungs than mice treated with the Standard Regimen, while mice treated with PRS Regimen IIC had 4.0 logs fewer lung CFU counts than Standard Regimen-treated mice. Both regimens were also markedly more efficacious than the Standard Regimen in achieving relapse-free cure. Whereas the Standard Regimen 

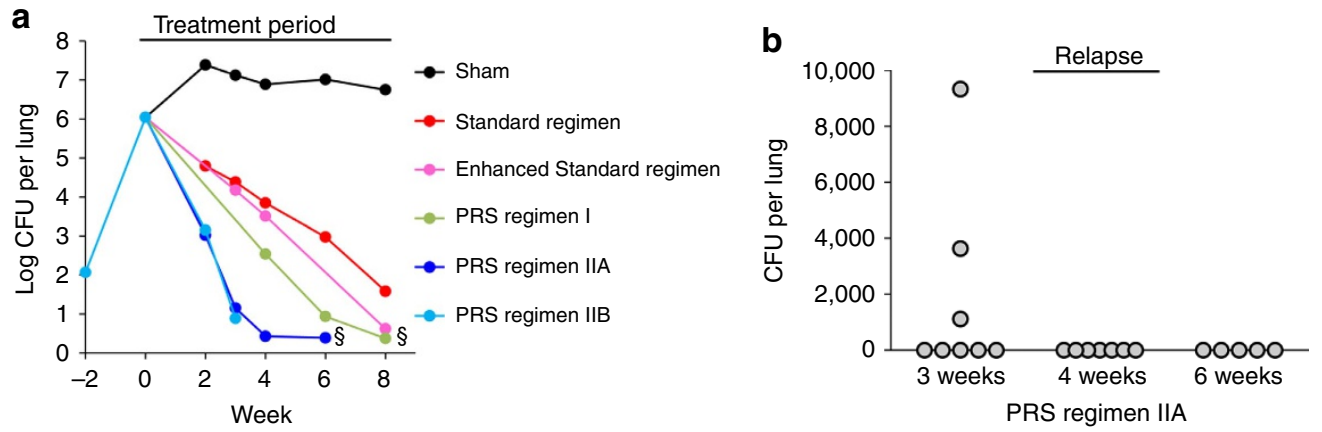

C
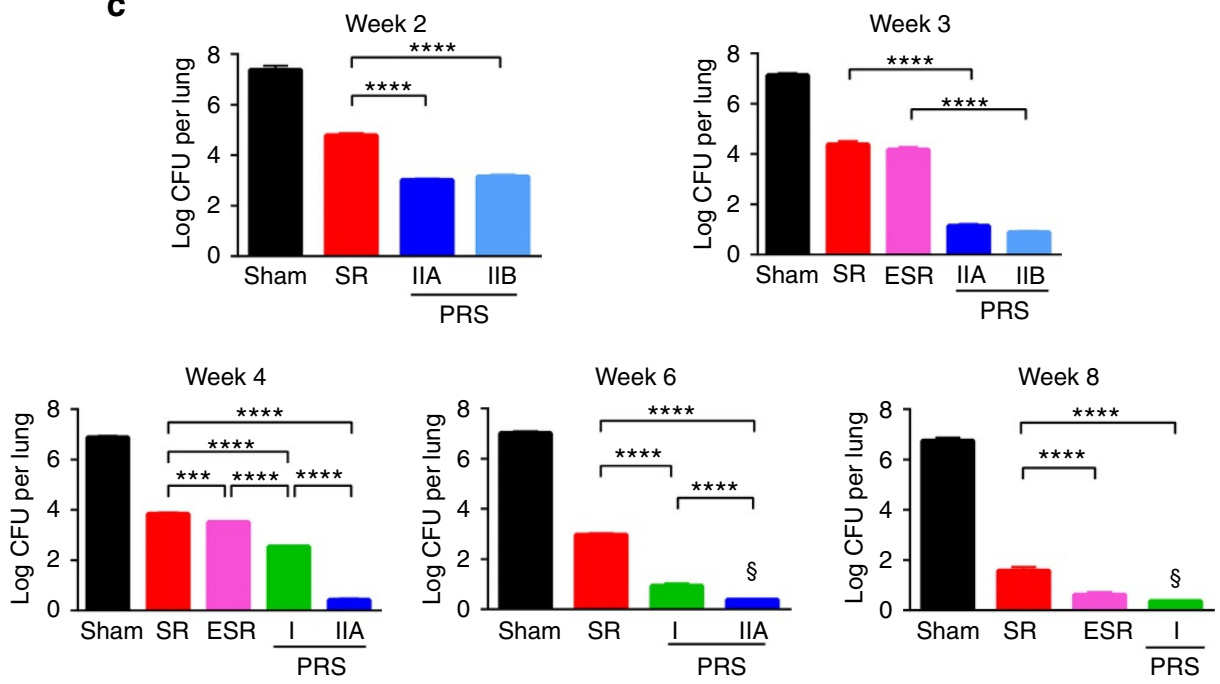

Figure 4 | Medium-term efficacy and relapse study. (a) M. tuberculosis burden in the lung over the course of infection and treatment period, where mice were sham-treated or treated with the Standard Regimen (SR), Enhanced Standard Regimen (ESR) or PRS Regimen I, IIA or IIB starting at Week 0. Data are mean $\log _{10}$ CFU for $n=5$ mice per group. ${ }^{\S} \mathrm{CFU}$ at limit of detection. (b) Relapse 3 months after completion of treatment with PRS Regimen IIA for the duration indicated. (c) M. tuberculosis lung burden after treatment 5 days per week for 2, 3, 4, 6 and 8 weeks in sham-treated mice or mice treated with Standard Regimen (SR), Enhanced Standard Regimen (ESR) or PRS Regimen I, IIA or IIB. Data are mean \pm s.e.m. of $\log _{10}$ CFU for $n=5$ mice per group. Differences in efficacy between groups were evaluated by one-way ANOVA with Tukey's correction. ${ }^{\star \star \star} P<0.001,{ }^{\star \star \star \star} P<0.0001$. ${ }^{\S}$ No M. tuberculosis CFU detected-data plotted as discussed in Methods.

required 12 weeks to achieve $80 \%$ relapse-free cure and 16 weeks to achieve $100 \%$ relapse-free cure in our in vivo model, PRS Regimen I achieved these milestones in 8 and 12 weeks, respectively, and PRS Regimen IIC in 3 and 4 weeks, respectively. Thus, compared with the Standard Regimen, PRS Regimens I and IIC reduced the duration of treatment required to achieve $80 \%$ relapse-free cure by 33 and $75 \%$, respectively, and $100 \%$ relapsefree cure by $25 \%$ and $75 \%$, respectively. In addition, as discussed below, PRS Regimen II achieves lung culture negativity and relapse-free cure in the mouse model faster than other regimens shown to accelerate treatment ${ }^{26-29}$. If our results in mice extrapolate to humans, PRS Regimen IIC would be expected to reduce the duration of treatment of TB from 6-8 months, as is currently the case for the Standard Regimen, to $1 \frac{112-2}{2}$ months. Moreover, as neither PRS Regimen I nor PRS Regimen II includes INH, RIF, fluoroquinolones or injectable aminoglycosides, these regimens should allow treatment, with a similar expedited time frame, of many cases of multidrug resistant TB (MDR-TB; defined as TB resistant to INH and RIF) and those cases of extensively drug-resistant TB (XDR-TB; defined as TB additionally resistant to fluoroquinolones and injectable aminoglycosides) in which susceptibility to PZA is preserved.

As others have done, we compared our PRS regimens to the Standard Regimen in the mouse model using the conventional mouse doses. While our PRS regimens were optimized in the mouse, the Standard Regimen was essentially optimized in humans and the mouse doses extrapolated from pharmacologic data. Nevertheless, the Standard Regimen extrapolated pharmacologically to mice is an important benchmark that is routinely used in mouse efficacy studies and provides an important reference point for comparisons between studies.

PRS Regimen II, in comparison with the Standard Regimen, reduces the time required to render the lung culture negative and achieve relapse-free cure by a greater amount (75\%) than several previously reported regimens in the mouse model. For example, the combination of RIF/PZA/Moxifloxacin reduced the time to relapse-free cure by $33 \%$ (6 months to 4 months) ${ }^{23}$, and the combination of INH/PZA/RIF/CLZ by $50 \%$ ( 6 months to 3 months $)^{26}$. Williams et al. included in their studies a drug combination of BDQ/PZA/Rifapentine/CLZ that is similar to our PRS Regimen II in that it shares 3 of the 4 drugs (they used Rifapentine instead of EMB; in addition, they used a PZA dose of $150 \mathrm{mg} \mathrm{kg}^{-1}$ rather than $450 \mathrm{mg} \mathrm{kg}^{-1}$ ) and observed relapse in $4 / 15(27 \%)$ mice after 4 weeks treatment and relapse in $0 / 15(0 \%)$ mice at 6 weeks ${ }^{22}$; the regimen was not compared with a standard regimen. In comparing even very similar regimens, however, it should be appreciated that because each drug regimen involves multiple potential positive and negative drug-drug interactions, that a change of just one drug in a multiple drug regimen may have a profound impact on efficacy. Rosenthal et al..$^{30}$ 
a

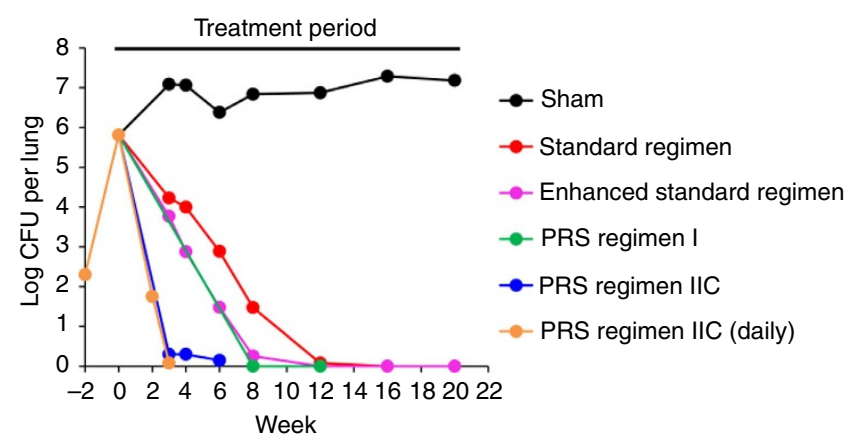

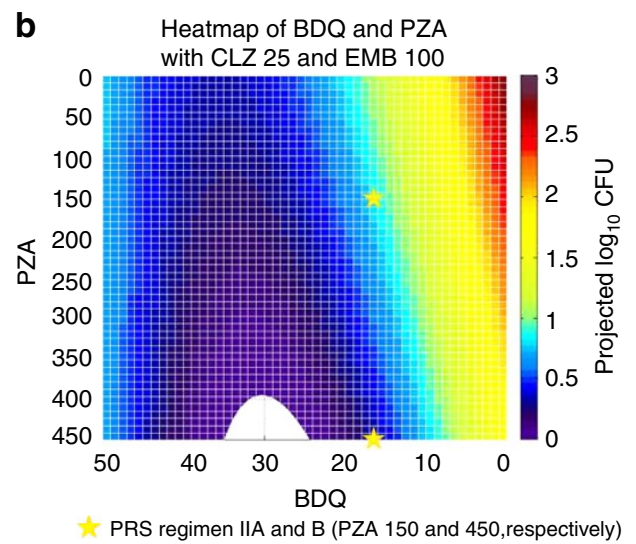

PRS regimen IIA and B (PZA 150 and 450,respectively)
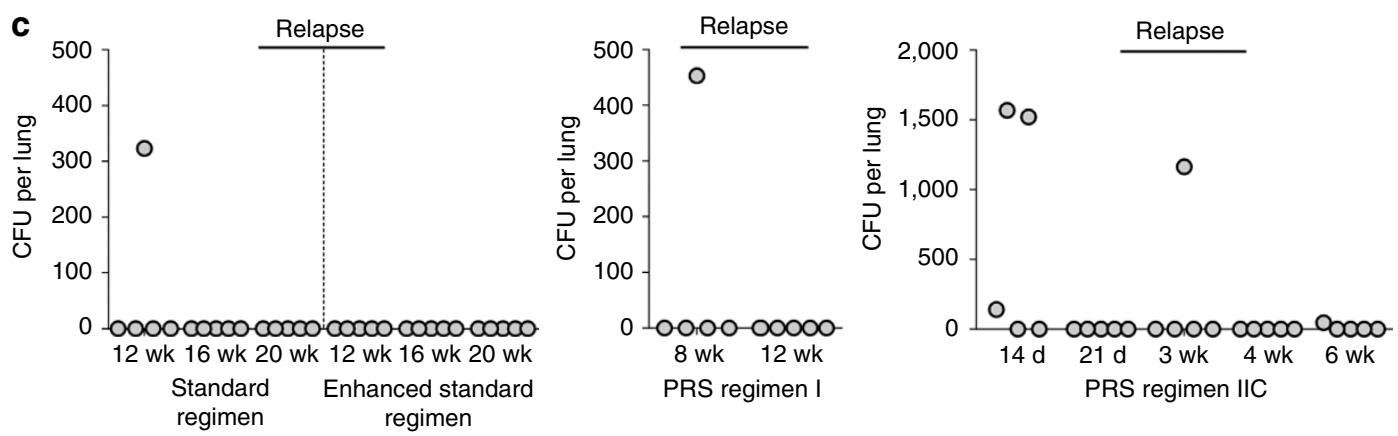

Figure 5 | Long-term efficacy and relapse study. (a) M. tuberculosis burden in the lung over the course of infection and treatment period, where mice were sham-treated or treated with the Standard Regimen, Enhanced Standard Regimen or PRS Regimen I or IIC 5 days (Monday-Friday) a week starting at Week 0. The PRS Regimen IIC (daily) group was treated daily for 14 days. Data transformation as $\log _{10}(x+1)$ with $x$ being the actual CFU was used for graphing purpose. (b) Heatmap for PRS Regimen II with CLZ and EMB dose at 25 and $100 \mathrm{mg} \mathrm{kg}^{-1}$, respectively, indicating that the optimal doses of $\mathrm{BDQ}$ and PZA were 30 and $450 \mathrm{mg} \mathrm{kg}^{-1}$, respectively. The white zone around BDQ $30 \mathrm{mg} \mathrm{kg}^{-1}$ at the bottom of the map corresponds to CFU projected to be 0 or below. (c) Relapse in the lung 3 months after completion of treatment with the PRS Regimen I or IIC or control regimens (Standard Regimen or Enhanced Standard Regimen) daily (d) or 5 days per week (wk) for the duration indicated. Differences in time to relapse-free cure between the Standard Regimen and the PRS regimens administered at the same frequency ( 5 days per week) were statistically significant ( $P=0.002$ versus PRS Regimen I and $P<0.0001$ versus PRS Regimen IIC, log rank test). Differences between PRS Regimens I and II in time to relapse-free cure (administered at the same frequency (5 days per week)) were also statistically significant $(P<0.0001, \log$ rank test).

observed that MXF/PZA $\left(150 \mathrm{mg} \mathrm{kg}^{-1}\right) /$ Rifapentine cleared bacteria in the lungs of mice after 2 months of treatment and reduced the time to relapse-free cure (0\% relapse) by $50 \%$ compared with INH/PZA $\left(150 \mathrm{mg} \mathrm{kg}^{-1}\right) / \mathrm{RIF}$, which required 6 months to achieve relapse-free cure in all of the mice.

So far, our studies in the mouse TB model have explored only two of the drug combinations that our in vitro PRS study identified as being substantially more effective than the Standard Regimen, and in both cases our in vivo mouse studies confirmed the predictions of our in vitro macrophage studies. However, while antimicrobial efficacy in macrophages is important and probably a pre-requisite for efficacy in vivo, the properties of the drug combinations that make them highly effective in vivo may differ from the properties that make them effective in macrophage cell culture, and it is vitally important to test and optimize the drug combinations both in vitro and in vivo. For example, whereas the macrophage cell cultures are bathed in a constant concentration of antibiotics, it is likely that pharmacokinetics plays a major role in efficacy in the mouse model and that the very long half-lives of CLZ and $\mathrm{BDQ}$ make them potent components in the combination cocktails.

All TB drugs can occasionally cause severe adverse effects, some readily manageable and others not; even when not manageable, most adverse effects are reversible upon drug discontinuation. With respect to drug toxicity, the Standard Regimen comprises three hepatotoxic drugs (INH, RIF, PZA), three drugs that can cause gastrointestinal intolerance (INH, RIF, PZA), two drugs that can cause optic neuritis (EMB, INH) and a drug that can lead to peripheral neuropathy (INH), among other adverse effects ${ }^{3,31-33}$. Both PRS regimens share two of these drugs with the Standard Regimen (EMB, PZA) and also include CLZ, which can cause gastrointestinal intolerance, prolong the QTc interval, and result in reversible skin discoloration (bronzing) ${ }^{33}$. In addition, PRS Regimen I includes PRO, which can cause gastrointestinal intolerance and be hepatotoxic ${ }^{33}$, while PRS Regimen II includes BDQ, which can prolong the QTc interval and be hepatotoxic ${ }^{34}$. All things considered, managing patients on the PRS regimens should be no more difficult and probably less difficult than managing patients on the Standard Regimen.

One of the interesting findings in our PRS in vitro studies was that many of the most promising drug combinations included CLZ. Although the anti-TB activity of CLZ was identified in the 1950s, it has not featured prominently in TB treatment regimens because it was initially thought to be poorly active in patients with TB. However, interest in CLZ has resurfaced consequent to the challenge of treating MDR- and XDR-TB. A CLZ-containing regimen (combined with gatifloxacin, EMB, and PZA, and supplemented with PRO, kanamycin and high-dose INH for an intensive phase of at least 4 months) was shown both to be highly effective for treatment of MDR-TB and to allow duration of therapy for MDR-TB to be shortened from 20 months (or more) to $9-12$ months ${ }^{27}$. Recent studies in a mouse model of TB have 
shown that addition of CLZ to a first-line regimen provides more rapid lung sterilization (3 versus 5 months) and shortens the duration of treatment needed to achieve relapse-free cure (3 versus 6 months) ${ }^{26}$. Similarly, addition of CLZ to a second-line drug regimen in a mouse model of $\mathrm{INH}$-resistant $\mathrm{TB}$ also provided more effective lung sterilization and provided relapsefree cure after 9 months, whereas in the absence of CLZ, the mouse lung tissue was not sterilized after 9 months of treatment ${ }^{28}$. As monotherapy, CLZ does not show early bactericidal activity in mice ${ }^{35}$ or $\operatorname{man}^{29}$ and it did not contribute to early bactericidal activity in combination with other drugs in human studies ${ }^{29}$. Nevertheless, it is likely that CLZ is beneficial in combination regimens both because its long half-life provides sustained antibiotic coverage and because of synergistic activity with other antibiotics. From the standpoint of synergistic mechanisms, Williams et al. observed that PZA and CLZ increased the bactericidal activity of BDQ in their mouse model of pulmonary TB and hypothesized that BDQ, PZA, and CLZ may have additive effects through inhibition of ATP synthesis and that the ATP depleting activity of CLZ and BDQ could prevent the efflux of pyrazinoic acid ${ }^{22}$.

In PRS Regimen I, whereas PRO and EMB showed a relatively flat dose-response relationship, increasing the PZA dose yielded substantial increases in efficacy. A similar strong dose-response relationship was demonstrated by Ahmad et al. ${ }^{24}$ for PZA both as monotherapy and in combination with RIF, prompting these authors to suggest clinical studies to evaluate whether the clinically used dose of PZA should be increased. Although lower PZA doses of $150-300 \mathrm{mg} \mathrm{kg}^{-1}$ are more often used in mouse studies, we found that the $450 \mathrm{mg} \mathrm{kg}^{-1}$ dose of PZA was well tolerated by the mice. A surface area-based conversion factor ('FDA Guidance for Industry: Estimating the Maximum Safe Starting Dose in Initial Clinical Trials for Therapeutics in Adult Healthy Volunteers', available at http://www.fda.gov/downloads/ Drugs/.../Guidances/UCM078932.pdf) predicts the mouse $450 \mathrm{mg} \mathrm{kg}^{-1}$ dose to be equivalent to a $36.6 \mathrm{mg} \mathrm{kg}^{-1}$ dose for a $60 \mathrm{~kg}$ human, and this dose is only slightly higher than the clinically recommended dose $\mathrm{e}^{27}$, just above the upper end of the $15-30 \mathrm{mg} \mathrm{kg}^{-1}$ dosing range recommended by the CDC-ATS guidelines $^{36}$. Comparison of published PZA PK data in mice and humans also suggest that the standard $150 \mathrm{mg} \mathrm{kg}^{-1}$ dose of PZA in mice yields a lower AUC than a human dose of $30 \mathrm{mg} \mathrm{kg}^{-1}$. In humans, an oral PZA dose of $27 \mathrm{mg} \mathrm{kg}^{-1}$ yielded an AUC of $520 \mathrm{mghl}^{-1}$ in one study ${ }^{37}$ and in another study a dose of $22-30 \mathrm{mg} \mathrm{kg}^{-1}$ yielded an AUC of $617-738 \mathrm{mg} \mathrm{hl}^{-1}$ (ref. 38). In contrast, an oral PZA dose of $150 \mathrm{mg} \mathrm{kg}^{-1}$ in BALB/c mice yielded significantly lower AUCs of $350 \mathrm{mghl}^{-1}$ (ref. 39) and $388 \mathrm{mg} \mathrm{h} \mathrm{1}^{-1}$ (ref. 38). Moreover, PZA doses of $50-60 \mathrm{mg} \mathrm{kg}^{-1}$ have been used clinically and a systematic review and metaanalysis determined that higher doses of PZA were not associated with any dose-dependent increase in hepatotoxicity ${ }^{40}$. Indeed, the concerns over PZA related hepatotoxicity have been in the setting in which it is used in the Standard Regimen in combination with the hepatotoxic drugs INH and RIF; thus, the Enhanced Standard Regimen tested in our mouse studies, where the PZA dose was substantially increased compared with the pharmacologically equivalent human dose and which showed improved efficacy compared with the Standard Regimen in the mouse, is not likely to extrapolate safely to humans. In contrast, PRS Regimens I and II do not include these additional two hepatotoxic drugs, making it possible to safely administer a substantially higher dose of PZA. While higher doses of PZA in the setting of PRS Regimens I and II could be associated with an increase in other side effects such as arthralgias and elevated uric acid levels, these are likely to be manageable and the shorter course of therapy would likely reduce the incidence of serious adverse events. Of note, however, while the higher dose of PZA may be ideal, for both PRS Regimens I and II, our drug dose-efficacy response surface model indicates that lower doses of PZA can be used without significantly compromising efficacy.

Our previous in vitro PRS studies employed PRO rather than ethionamide (ETA) because, although the two drugs are considered interchangeable, PRO has been found to outperform ETA in a clinical trial of leprosy and to be better tolerated than ETA (ref. 33). Nevertheless, we anticipate that ETA could replace PRO in our PRS regimens without loss of efficacy.

PRS I Regimen I (CLZ/EMB/PRO/PZA) has the advantage of being inexpensive and these drugs are readily available on the formulary in most countries. On the basis of our mouse studies, we predict that PRS Regimen II (CLZ/EMB/BDQ/PZA) will provide even more rapid bacterial clearance in the tissues and will achieve relapse-free cure even sooner.

Whether our mouse model will accurately predict the results in humans remains to be determined. There are reasons why the model may overestimate or underestimate the efficacy of various drug combinations. As others have noted ${ }^{22}$, some differences between TB in mouse and human could lead the BALB/c model to overestimate the clinical efficacy of BDQ and CLZ. For example, unlike humans, the BALB/c mouse does not develop cavitary disease and the bacteria are almost exclusively intracellular. Since CLZ and BDQ are very hydrophobic, they become concentrated intracellularly and might be less effective against extracellular TB (ref. 22). Consistent with this, Irwin et $a l^{41}$ recently observed that CLZ showed much greater TB killing activity in $\mathrm{BALB} / \mathrm{c}$ mice than in $\mathrm{C} 3 \mathrm{HeB} / \mathrm{FeJ}$ mice that develop caseous necrosis with numerous extracellular TB. It is possible that the duration of treatment may require adjustment depending on the presence or absence of cavitary disease. On the other hand, the mouse, which does not develop as strong a cell-mediated immune response as humans, as reflected by less organized granulomata and relatively weak cutaneous delayedtype hypersensitivity responses ${ }^{42}$, may underestimate the clinical efficacy of drugs at achieving relapse-free cure. Our studies indicate that as few as 1 or 2 bacteria remaining in the lung at the cessation of treatment are sufficient to allow relapse in our mouse model.

Our studies demonstrate that systematic in vitro and in vivo PRS methodology can identify and optimize drug combinations for treatment of TB that are substantially more effective than the current Standard Regimen. Expanding the PRS methodology to additional drug combinations, including some of the newly developed drugs, has the potential to identify combinations that are even more potent.

\section{Methods}

Aerosol infection. Eight-week old, female, pathogen-free BALB/c mice were obtained from Taconic, housed in groups of five, and provided with unlimited access to food and water. Mice were challenged by aerosol with the highly virulent M. tuberculosis Erdman strain (ATCC 35801). One day later, two mice were killed to determine the initial number of CFU implanted in their lungs. After two weeks, three mice were killed to determine the number of CFU in the lungs at the start of treatment.

Drug treatment. Two weeks after infection, mice were treated by oral gavage five times per week (Monday-Friday) for 3-24 weeks, or sometimes daily for 2-3 weeks. Mice under treatment with the Standard Regimen were first administered EMB, INH, and PZA together in $0.1 \mathrm{ml}$ of $0.15 \%$ agarose suspension, and $1 \mathrm{~h}$ later administered RIF in water. Mice under treatment with PRS regimens were first administered CLZ alone in $0.1 \mathrm{ml}$ of $0.15 \%$ agarose suspension and $1 \mathrm{~h}$ later administered EMB/PRO/PZA (PRS Regimen I) or EMB/BDQ/PZA (PRS Regimen II) together in $0.1 \mathrm{ml} 0.15 \%$ agarose suspension. Sham-treated mice were first administered agarose suspension and $1 \mathrm{~h}$ later administered water. CLZ, EMB, INH, PRO, RIF and PZA were purchased from Sigma-Aldrich. BDQ was provided by the Global Alliance for TB Drug Development. Drug doses used in this study are listed in Table 1. 
Assessment of treatment bactericidal activity. Mice were killed three days after the last treatment dose. Their lung, liver and spleen were homogenized in $1 \mathrm{ml}$ of phosphate-buffered saline. The homogenates were serially diluted and spread $100 \mu \mathrm{l}$ per plate on $7 \mathrm{H} 11$ agar containing $0.4 \%$ activated charcoal, ampicillin $\left(12.5 \mu \mathrm{g} \mathrm{ml}^{-1}\right)$, amphotericin $\left(5 \mu \mathrm{g} \mathrm{ml}^{-1}\right)$ and polymyxin B $\left(20 \mathrm{Uml}^{-1}\right)$. The plates were incubated at $37^{\circ} \mathrm{C}$ in a $5 \% \mathrm{CO}_{2}-95 \%$ air atmosphere for 31 days, after which the number of CFU of $M$. tuberculosis on each plate was enumerated. The limit of detection for CFU ranged from 4.4 to 5 organisms per lung depending on the total volume of lung homogenate from each animal. When no organism was detected on any of the plates, the CFU per lung was plotted as half of the limit of detection. When only a single colony was detected on all of the plates, the CFU per lung was plotted as the limit of detection.

Assessment of relapse. Mice were held for 3 months after the last treatment; killed; the lung and spleen homogenized; and the entire homogenate plated on 60 (20 for each lung and 40 for each spleen) charcoal 7H11 agar plates. CFU on the plates was determined after incubation for 31 days at $37^{\circ} \mathrm{C}$ in a $5 \% \mathrm{CO}_{2}-95 \%$ air atmosphere. The absence of a single $\mathrm{CFU}$ in the entire lungs of mice 3 months after treatment cessation was considered relapse-free cure.

Animal studies. All animal studies were approved and conducted in accordance with the procedures as set forth by the UCLA Animal Research Committee. The BALB/c mouse strain was used as this is the established model for studies of the efficacy of multidrug TB regimens ${ }^{10,22,23,26,28,30,35,43-46}$. Experience has shown that the results of multidrug efficacy studies in the mouse model of pulmonary tuberculosis are generally, albeit not perfectly, predictive of their efficacy in humans ${ }^{10-12}$.

Parabolic drug dose-efficacy response surface. In a previous study ${ }^{8}$, we identified highly promising drug combinations for killing M. tuberculosis in human macrophages. Here, we evaluate two of these combinations (PRS Regimens I and II), each consisting of 4 drugs, in vivo in the mouse model of pulmonary tuberculosis. To transition from in vitro to in vivo studies, we first needed to identify the optimal doses of these drugs in each PRS regimen in vivo. This was accomplished using PRS methodology ${ }^{8}$, which in essence describes the relationship between a phenotypic response in animals (the output measurement, in this case the lung burden of $M$. tuberculosis) and doses of different drugs used to effect this phenotypic response. Based upon our previous findings in multiple complex biological settings, including in vitro studies involving eukaryotic cells, in vivo studies, and clinical studies ${ }^{7-19,47-49}$, the relationship between the phenotypic response and the doses of drugs used to effect the phenotypic response fits a parabolic response surface (sometimes referred to herein as the drug dose-efficacy response surface). Such a surface can be described by a second order algebraic (quadratic) equation (see below), as opposed to much more complex surfaces requiring, for example, third and fourth order algebraic equations to describe them. A major advantage of the PRS approach is that a relatively small number of tests is required to determine the coefficients of the second order algebraic equation and thus define the parabolic response surface. In the current study, the drug combinations each contained 4 drugs; ordinarily the second order algebraic equation for four drugs would have 15 coefficients, which can be determined by 15 tests (or in this case groups of animals receiving different drug-dose combinations). By keeping the dose of one drug (CLZ) in each combination constant, we reduced the number of tests required to 10 , the number of coefficients in the second order algebraic equation for 3 drugs; this allowed substantial savings in cost, labour, and time. Thus, only 10 groups of animals, where each group was treated with a different dose ratio of the three drugs, were needed to map out the drug-dose efficacy response surface. We chose CLZ as the drug to be kept at a constant dose because CLZ has an extraordinarily long half-life ${ }^{35}$. Moreover, Swanson et al. have recently shown in a mouse model of TB that CLZ exhibits a threshold effect, such that differences in CLZ concentrations in the blood and tissues do not translate into dose-dependent differences in antimicrobial activity ${ }^{35}$.

Specifically, in this study, the lung burden of M. tuberculosis $\left(\log _{10} \mathrm{CFU}\right)$ in mice after treatment with PRS Regimen I or II drugs at various drug doses was used to generate the second-order (parabolic) algebraic model using MATLAB LinearModel.stepwise function (Mathworks Inc., Natick, MA); and the correlation between experimental and modelled $\log _{10} \mathrm{CFU}$ was determined using the MATLAB linear or rank correlation function. The parabolic response surface is expressed as:

$y=\beta_{0}+\beta_{1} x_{1}+\ldots+\beta_{n} x_{n}+\beta_{12} x_{1} x_{2}+\ldots+\beta_{m n} x_{m} x_{n}+\beta_{11} x_{1}^{2}+\ldots+\beta_{n n} x_{n}^{2}+\mathrm{e}$

where $y$ represents the log CFU; $x_{n}$ is the $n$th drug dosage; $\beta_{0}$ is the intercept term; $\beta_{n}$ is the single drug coefficient of the $n$th drug; $\beta_{m n}$ is the interaction coefficient between the $m$ th and $n$th drugs; $\beta_{n n}$ is the quadratic coefficient for the $n$th drug; and $\mathrm{e}$ is the residual error assumed to have a normal distribution with constant s.d. $=\sigma_{\mathrm{e}}$.

As noted above, in determining the parabolic response surface, by keeping the dose of one drug in the combination constant, we reduced the number of unknown coefficients $(\beta \mathrm{s})$ in equation (1) above to 10 . Therefore, in this study, $n=3$ in equation (1). We applied the Orthogonal Array Composite Designs (OACD) method to place the 10 drug-dose combinations (experimental groups) at strategic positions for locating the most accurate parabolic response surface ${ }^{15,16}$. This was done by selecting high $(\mathrm{H})$, medium $(\mathrm{M})$ and low $(\mathrm{L})$ drug doses of each drug and ensuring that each pair of conditions (for example, HH, HM, HL, MH, MM, ML, LH, LM and LL) for any two drugs occurred equally often for all of the drug pairs in the combinations being tested. The use of an orthogonal array test design to map the parabolic response surface allowed us to maximize our coverage of the drug dose-efficacy response surface, while minimizing the number of combinations to be tested.

A parabolic regression model was fit to the $\log _{10}$ CFU data using equation (1) above, giving the expected $\log _{10} \mathrm{CFU}$ as a function of the drug dose combinations. For every observed $\log _{10} \mathrm{CFU}$, a corresponding predicted $\log _{10} \mathrm{CFU}$ value was generated by this equation. The residual errors (e) were defined as the difference between the observed $\log _{10}$ CFU and the predicted $\log _{10} \mathrm{CFU}\left(\mathrm{e}=\right.$ observed $\log _{10}$ CFU-predicted $\log _{10} \mathrm{CFU}$ ). A normal quantile plot of the residual errors confirmed that they followed a normal distribution.

In determining the final parabolic response surface, we removed non-significant terms from the original 10 term equation using a stepwise hierarchical removal rule $^{50}$ and a $p<0.05$ term retention criterion. For PRS Regimen I, the parabolic drug dose-efficacy response surface determined from the lung $\log _{10}$ CFU data is given in equation (2), below:

$$
\begin{aligned}
\log _{10} \mathrm{CFU}= & 3.3633+(0.0017925)(\mathrm{EMB})+(0.0063399)(\mathrm{PRO}) \\
& +(0.0018712)(\mathrm{PZA})-\left(8.4882 \times 10^{-6}\right)(\mathrm{EMB})(\mathrm{PZA}) \\
& -\left(2.2351 \times 10^{-5}\right)(\mathrm{PRO})(\mathrm{PZA})-\left(6.1296 \times 10^{-6}\right)\left(\mathrm{PZA}^{2}\right),
\end{aligned}
$$

where $\mathrm{EMB}, \mathrm{PRO}$ and $\mathrm{PZA}$ represent the dose of $\mathrm{EMB}, \mathrm{PRO}$ and $\mathrm{PZA}$, respectively, in $\mathrm{mg} \mathrm{kg}^{-1}$.

For PRS Regimen II, the parabolic drug dose-efficacy response surface determined from the lung $\log _{10} \mathrm{CFU}$ data is given in equation (3), below:

$$
\begin{aligned}
\log _{10} \mathrm{CFU}= & 2.7846-(0.13892)(\mathrm{BDQ})-(0.0024415)(\mathrm{PZA}) \\
& +\left(4.7592 \times 10^{-5}\right)(\mathrm{BDQ})(\mathrm{PZA})+(0.0019807)\left(\mathrm{BDQ}^{2}\right),
\end{aligned}
$$

where BDQ and PZA represent the dose of BDQ and PZA, respectively, in $\mathrm{mg} \mathrm{kg}^{-1}$.

Once the parabolic drug dose-efficacy response surface was obtained from the experimental data, the optimal drug doses were derived as those giving the lowest projected mean number of $M$. tuberculosis $\left(\log _{10} \mathrm{CFU}\right)$ in the mouse lung.

Statistics. On the basis of previous studies ${ }^{10,28}$ showing that mean differences in $\log _{10}$ CFU were 2 s.d. or larger, we chose a sample size of 5 mice per group as it provided $80 \%$ power to confirm mean differences of 2 s.d. or larger using the $P<0.05$ significance criterion. Per cent relapse versus treatment time were compared across groups using the log rank test since this data did not follow the normal distribution and most observations were censored (that is the animal did not relapse). Means and s.e. of the mean (s.e.m.) are reported. Data collected from the animal studies were analysed using GraphPad Prism software (version 6.05). Means were compared across groups by ANOVA with Tukey's correction for multiple comparisons. Levene's test was used to assess error homoscedasticity for both the parabolic model and the ANOVA.

Additional statistical analyses of the parabolic drug dose-efficacy response surface model are described in the preceding section entitled Parabolic drug dose-efficacy response surface.

Code availability. The functions used for parabolic curve fitting to obtain the coefficients of the second order algebraic equation and for statistical analysis are available in MATLAB. An example of the MATLAB code used for parabolic curve fitting so as to obtain the coefficients of the second order algebraic equation and for statistical analysis is provided as Supplementary Software.

Data availability. All data generated or analysed during this study are included in this published article and its supplementary information files, or are available from the corresponding author on request.

\section{References}

1. World Health Organization. Global Tuberculosis Report. Available at http:// www.who.int/tb/publications/global_report/en/ (2015).

2. Center for Disease Control. Treatment for TB Disease. Available at https:// www.cdc.gov/tb/topic/treatment/tbdisease.htm (2016).

3. World Health Organization. Treatment of Tuberculosis: Guidelines 4th edn. Available at http://www.who.int/tb/publications/2010/9789241547833/en/ (2010).

4. Zumla, A., Nahid, P. \& Cole, S. T. Advances in the development of new tuberculosis drugs and treatment regimens. Nat. Rev. Drug Discov. 12, 388-404 (2013).

5. Zumla, A. et al. Tuberculosis treatment and management-an update on treatment regimens, trials, new drugs, and adjunct therapies. Lancet Resp. Med. 3, 220-234 (2015). 
6. Nuermberger, E. L., Spigelman, M. K. \& Yew, W. W. Current development and future prospects in chemotherapy of tuberculosis. Respirology 15, 764-778 (2010).

7. Ma, Z., Lienhardt, C., McIlleron, H., Nunn, A. J. \& Wang, X. Global tuberculosis drug development pipeline: the need and the reality. Lancet $\mathbf{3 7 5}$, 2100-2109 (2010).

8. Silva, A. et al. Output-driven feedback system control platform optimizes combinatorial therapy of tuberculosis using a macrophage cell culture model. Proc. Natl Acad. Sci. USA 113, E2172-E2179 (2016).

9. Grosset, J. \& Ji, B. in Mycobacteria: II. Chemother. 51-97 (Chapman and Hall, 1998).

10. Nuermberger, E. L. et al. Moxifloxacin-containing regimen greatly reduces time to culture conversion in murine tuberculosis. Am. J. Respir. Crit Care Med. 169, 421-426 (2004)

11. Li, S. Y. et al. Evaluation of moxifloxacin-containing regimens in pathologically distinct murine tuberculosis models. Antimicrob. Agents Chemother. 59, 4026-4030 (2015).

12. Nuermberger, E. Using animal models to develop new treatments for tuberculosis. Semin Respir. Crit Care Med. 29, 542-551 (2008).

13. Wong, P. K. et al. Closed-loop control of cellular functions using combinatory drugs guided by a stochastic search algorithm. Proc. Natl Acad. Sci. 105, 5105-5110 (2008).

14. Al-Shyoukh, I. et al. Systematic quantitative characterization of cellular responses induced by multiple signals. BMC Syst. Biol. 5, 88 (2011).

15. Ding, X., Xu, H., Hopper, C., Yang, J. \& Ho, C.-M. Use of fractional factorial designs in antiviral drug studies. Qual. Reliab. Eng. Int. 29, 299-304 (2013).

16. Jaynes, J., Ding, X., Xu, H., Wong, W. K. \& Ho, C. M. Application of fractional factorial designs to study drug combinations. Stat. Med. 32, 307-318 (2013).

17. Ding, X. et al. Discovery of a low order drug-cell response surface for applications in personalized medicine. Phys. Biol. 11, 065003 (2014).

18. Wang, H. et al. Mechanism-independent optimization of combinatorial nanodiamond and unmodified drug delivery using a phenotypically driven platform technology. ACS Nano 9, 3332-3344 (2015).

19. Weiss, A. et al. A streamlined search technology for identification of synergistic drug combinations. Sci. Rep. 5, 14508 (2015).

20. Ding, X. et al. Cascade search for HSV-1 combinatorial drugs with high antiviral efficacy and low toxicity. Int. J. Nanomed. 7, 2281-2292 (2012)

21. Lefford, M. J. in The Mycobacteria: A Sourcebook (eds Kubica, G. P. \& Wayne, L. G.) 947-977 (Dekker, 1984).

22. Williams, K. et al. Sterilizing activities of novel combinations lacking first- and second-line drugs in a murine model of tuberculosis. Antimicrob. Agents Chemother. 56, 3114-3120 (2012).

23. Nuermberger, E. L. et al. Moxifloxacin-containing regimens of reduced duration produce a stable cure in murine tuberculosis. Am. J. Respir. Crit Care Med. 170, 1131-1134 (2004).

24. Ahmad, Z. et al. Dose-dependent activity of pyrazinamide in animal models of intracellular and extracellular tuberculosis infections. Antimicrob. Agents Chemother. 55, 1527-1532 (2011).

25. Cynamon, M. H. \& Sklaney, M. Gatifloxacin and ethionamide as the foundation for therapy of tuberculosis. Antimicrob. Agents Chemother. 47, 2442-2444 (2003).

26. Tyagi, S. et al. Clofazimine shortens the duration of the first-line treatment regimen for experimental chemotherapy of tuberculosis. Proc. Natl Acad. Sci. 112, 869-874 (2015).

27. Van Deun, A. et al. Short, highly effective, and inexpensive standardized treatment of multidrug-resistant tuberculosis. Am. J. Respir. Crit. Care Med. 182, 684-692 (2010).

28. Grosset, J. H. et al. Assessment of clofazimine activity in a second-line regimen for tuberculosis in mice. Am. J. Respir. Crit. Care Med. 188, 608-612 (2013).

29. Diacon, A. H. et al. Bactericidal activity of pyrazinamide and clofazimine alone and in combinations with pretomanid and bedaquiline. Am. J. Respir. Crit. Care Med. 191, 943-953 (2015)

30. Rosenthal, I. M. et al. Daily dosing of rifapentine cures tuberculosis in three months or less in the murine model. PLOS Med. 4, e344 (2007).

31. Saukkonen, J. J. et al. An official ATS statement: hepatotoxicity of antituberculosis therapy. Am. J. Respir. Crit Care Med. 174, 935-952 (2006).

32. Denholm, J. T. et al. Adverse effects of isoniazid preventative therapy for latent tuberculosis infection: a prospective cohort study. Drug Healthcare Patient Saf. 6, 145-149 (2014)

33. Global Alliance for TB Drug Development. Handbook of anti-tuberculosis agents. Tuberculosis (Edinb) 88, 85-86 (2008).

34. Fox, G. J. \& Menzies, D. A review of the evidence for using bedaquiline (TMC207) to treat multi-drug resistant tuberculosis. Infectious Diseases Therapy 2, 123-144 (2013).

35. Swanson, R. V. et al. Pharmacokinetics and pharmacodynamics of clofazimine in a mouse model of tuberculosis. Antimicrob. Agents Chemother. 59, 3042-3051 (2015)

36. Bass, Jr J. B. et al. Treatment of tuberculosis and tuberculosis infection in adults and children. American Thoracic Society and the Centers for Disease Control and Prevention. Am. J. Respir. Crit. Care Med. 149, 1359-1374 (1994).
37. Ahmad, Z. et al. Comparison of the 'Denver regimen' against acute tuberculosis in the mouse and guinea pig. J. Antimicrob. Chemother. 65, 729-734 (2010).

38. Via, L. E. et al. Host-mediated bioactivation of pyrazinamide: implications for efficacy, resistance, and therapeutic alternatives. ACS Infect. Dis. 1, 203-214 (2015).

39. Williams, K. N. et al. Addition of PNU-100480 to first-line drugs shortens the time needed to cure murine tuberculosis. Am. J. Respir. Crit. Care Med. 180, 371-376 (2009).

40. Pasipanodya, J. G. \& Gumbo, T. Clinical and toxicodynamic evidence that high-dose pyrazinamide is not more hepatotoxic than the low doses currently used. Antimicrob. Agents Chemother. 54, 2847-2854 (2010).

41. Irwin, S. M. et al. Limited activity of clofazimine as a single drug in a mouse model of tuberculosis exhibiting caseous necrotic granulomas. Antimicrob. Agents Chemother. 58, 4026-4034 (2014).

42. Lefford, M. J. in The Mycobacteria: A Sourcebook, Vol. 15 (eds Kubica, G.P. \& Wayne, L.G.) 947-977 (Dekker, 1984).

43. Lu, Y. et al. Clofazimine analogs with efficacy against experimental tuberculosis and reduced potential for accumulation. Antimicrob. Agents Chemother. 55, 5185-5193 (2011).

44. Tasneen, R. et al. Sterilizing activity of novel TMC207- and PA-824-containing regimens in a murine model of tuberculosis. Antimicrob. Agents Chemother. 55, 5485-5492 (2011).

45. Nuermberger, E. et al. Powerful bactericidal and sterilizing activity of a regimen containing PA-824, moxifloxacin, and pyrazinamide in a murine model of tuberculosis. Antimicrob. Agents Chemother. 52, 1522-1524 (2008).

46. Tyagi, S. et al. Bactericidal activity of the nitroimidazopyran PA-824 in a murine model of tuberculosis. Antimicrob. Agents Chemother. 49, 2289-2293 (2005).

47. Mohd Abdul Rashid, M. B. et al. Identification and optimization of combinatorial glucose metabolism inhibitors in hepatocellular carcinomas. J. Lab Autom. 20, 423-437 (2015).

48. Weiss, A. et al. Rapid optimization of drug combinations for the optimal angiostatic treatment of cancer. Angiogenesis 18, 233-244 (2015).

49. Zarrinpar, A. et al. Individualizing liver transplant immunosuppression using a phenotypic personalized medicine platform. Sci. Transl. Med. 8, 333ra349 (2016).

50. Glantz, S. \& Slinker, B. Primer of Applied Regression and Analysis of Variance 2nd edn (McGraw-Hill, 2001).

\section{Acknowledgements}

This work was supported by a subgrant from Shanghai Jiao Tong University, a grantee (Global Health Grant No. OPP1070754) of the Bill and Melinda Gates Foundation. We thank Jacques Grosset and Eric Nuermberger at Johns Hopkins University for helpful advice on preparation of orally administered antibiotics and Jeffrey Gornbein at University of California, Los Angeles for assistance with statistical analyses.

\section{Author contributions}

B.-Y.L., D.L.C., A.S., C.-M.H. and M.A.H. designed research; B.-Y.L., D.L.C., B.J.D., S.M.-G. and S.N. performed research; B.-Y.L., D.L.C., A.S., X.D., C.-M.H., M.A.H. analysed data; B.-Y.L., D.L.C. and M.A.H. wrote the paper.

\section{Additional information}

Supplementary Information accompanies this paper at http://www.nature.com/ naturecommunications

Competing financial interests: The authors are inventors on patents (International Patent Application Serial No. PCT/US2014/012111 and PCT/US2015/058892) covering the technology described herein.

Reprints and permission information is available online at http://npg.nature.com/ reprintsandpermissions/

How to cite this article: Lee, B.-Y. et al. Drug regimens identified and optimized by output-driven platform markedly reduce tuberculosis treatment time. Nat. Commun 8, 14183 doi: 10.1038/ncomms14183 (2017)

Publisher's note: Springer Nature remains neutral with regard to jurisdictional claims in published maps and institutional affiliations.

This work is licensed under a Creative Commons Attribution 4.0 International License. The images or other third party material in this article are included in the article's Creative Commons license, unless indicated otherwise in the credit line; if the material is not included under the Creative Commons license, users will need to obtain permission from the license holder to reproduce the material. To view a copy of this license, visit http://creativecommons.org/licenses/by/4.0/

(C) The Author(s) 2017 